\section{Levels of Immunoglobulin A1 in Peri- Implant Fluid and Saliva from Patients with Mucositis: A Preliminary Study}

\author{
Camilla Christian Gomes Moura ${ }^{1}$, Priscilla Barbosa Ferreira Soares ${ }^{2}$, Andreia \\ Espíndola Vieira ${ }^{3}$, Maria Aparecida de Souza ${ }^{4}$, Paula Dechichi ${ }^{3}$
}

'Department of Operative Dentistry and Dental Materials; ${ }^{2}$ Department of Periodontology and Implantology; ${ }^{3}$ Department of Morphology, School of Dentistry, UFU - Federal University of Uberlândia, Uberlândia, MG, Brazil ${ }^{4}$ Department of Immunology and Parasitology, Institute of Biomedical Sciences, UFU - Federal University of Uberlândia, Uberlândia, MG, Brazil

Correspondence: Dra. Camilla Christian Gomes Moura, Avenida República do Piratini, S/N, Bloco 4L, Anexo A, Sala 4 LA, 38400-902 Uberlândia, MG, Brasil. Tel: +55-34-32182255. Fax: +55-34-3218-2279. e-mail: pbfsoares@yahoo.com.br

Key Words: immunoglobulin $A$, saliva, dental implants, inflammation.

\section{Introduction}

The predominant immunoglobulin of mucosal secretions is $\lg A(1)$, which is divided into two subclasses, $\lg A 1$ and $\lg A 2$ (2-4). The IgA 1 predominates in serum, teeth and implants, and is a compound of sulcular fluid $(3,5)$, while $\lg A 2$ is found in higher concentrations in external secretions like saliva $(1,5)$. Both IgAs are found in saliva and sulcular fluid due to the intimate contact between the secretions $(2,3,5,6)$. The high levels of salivary $\lg A$ might protect against the development of gingivitis $(1,5)$. In the same way, IgA in sulcular fluid seems to have a protective function (4), which may be related to the lack of complement activation $(1,3)$.

The similarities between teeth sulcular fluid and periimplant sulcular have led to research evaluating the same biological markers present around teeth in order to analyze peri-implant sites $(7,8-10)$. These markers provide more sensitive and reliable tool than clinical or radiographic exams when diagnosing and monitoring the progression of the disease around teeth and implants $(8,11-13)$.

Once peri-implant inflammation is reflected in changes in the peri-implant sulcular fluid composition, which is a serum-like fluid exudate secreted by the gingival capillaries (10), changes in IgA1 levels could indicate inflammation. Therefore, IgA1 seems to be a possible diagnostic marker to evaluate the presence of peri-implant disease and to identify patients at risk of peri-implantitis. However, until this moment, there have been no studies evaluating the role of IgA 1 in saliva and in PISF when considering the degree of inflammation in a peri-implant site. Thus, the aim of the present study was to evaluate the $\lg A 1$ levels in the peri-implant sulcular fluid and saliva of partially edentulous patients to determine the potential use of this immunoglobulin as an early indicator of peri-implant disease, and evaluate whether IgA1 levels around implants are associated with salivary levels and with mucosa inflammation.

\section{Material and Methods \\ Patient Population}

Twenty-seven partially edentulous patients from a private clinic who had dental implants varying in length (from 10 to $15 \mathrm{~mm}$ ) and diameter (range of 3.3-5 mm) were included in this study. All implants had been installed by the same surgeon and were in function for at least 1 year. Criteria for patient selection were absence of systemic diseases, no antibiotics taken for at least 3 months before sampling, no smoking, no history of periodontal or periimplant therapy for at least 3 months and no history of medication that could interfere with saliva secretion. One implant was randomly selected from each patient for collection of the fluid samples. The protocol for collecting the samples and examining the patients was approved by the Ethics Committee of the Federal University of Uberlândia (Protocol \#181/06).

\section{Clinical Examination}

Clinical status of dental implant sites were evaluated by assessing the probing depth (PD) and bleeding on 
probing (BOP). Clinical measurements were taken at 6 sites: disto-buccal (DB), center-buccal (CB), mesio-buccal $(\mathrm{MB})$, disto-lingual (DL), center-lingual $(\mathrm{CL})$ and mesiolingual (ML) (8). PD was recorded from each site using a conventional periodontal probe (Hu-Friedy, Chicago, IL, USA). All measurements were performed after peri-implant sulcular fluid (PISF) sampling. Care was taken not to cause any physical injuries inside the sulcular area. Clinical examinations were performed by a single examiner to avoid inter-examiner variation.

\section{Criteria for Healthy and Diseased Peri-Implant Sites}

Patients were classified based on PD and BOP as either patients with peri-implant inflammation (mucositis) or healthy patients. The scores utilized for BOP were 0 , no bleeding when periodontal probe is passed along the gingival margin, and 1, bleeding on gingival margin. PD was categorized as I $=\leq 3 \mathrm{~mm}$; II = > $3 \mathrm{~mm} \leq 5 \mathrm{~mm}$; III $=>5 \mathrm{~mm}$. According to these definitions and analyzed parameters, a clinical diagnosis was applied as shown in Table 1.

\section{Sulcular Fluid Collection}

After removing supragingival plaque, the site was isolated with cotton rolls (Cremer, Blumenau, SC, Brazil) and dried with a gentle stream of air. Fluid samples were collected from the $D B, C B, M B, D L, C L$ and $M L$ aspects of each implant and tooth using a standardized endodontic paper (14), size 30 (Dentsply Maillefer, Ballaigues, Switzerland). The endodontic paper (Dentsply Maillefer) was inserted gently into the gingival sulcus until slight resistance was felt and then left in place for $30 \mathrm{~s}$. Samples containing blood were discarded. The endodontic papers (Dentsply Maillefer) from the same patient were placed into $1.5 \mathrm{~mL}$ plastic tubes (Eppendorf, Hamburg, Germany) containing $300 \mu \mathrm{L}$ of elution buffer (50 mM Tris- $\mathrm{HCl}$ buffer, $\mathrm{pH}$ 7.5, containing $0.15 \mathrm{M} \mathrm{NaCl}$ and $1 \mathrm{mM} \mathrm{CaCl}_{2}$ ).

In order to extract the sulcular fluid collected on the endodontic paper (Dentsply Maillefer), each sample tube was centrifuged twice at $10.000 \mathrm{~g}$ for $300 \mathrm{~s}$, the endodontic papers (Dentsply Maillefer) were discarded and the eluates stored at $-20^{\circ} \mathrm{C}$ prior to analysis. Therefore, from each patient was obtained one sample tube containing six

Table 1. Parameters used to implant classification

\begin{tabular}{lcc}
\hline & Probing depth & Bleeding on probing \\
\hline Healty group & $\leq 3$ & 0 \\
Mucositis group & $>3^{*}$ & $1^{*}$ \\
\hline
\end{tabular}

*at least one site showing probing depth $>3 \mathrm{~mm}$ and bleeding on probing. endodontic papers (Dentsply Maillefer), one wet paper per face $(D B, C B, M B, D L, C L$ and $M L)$.

\section{Saliva Samples}

Unstimulated whole saliva specimens were obtained in the morning prior to clinical evaluation. The nearest five milliliters of whole saliva were collected after the subjects were instructed to allow saliva to pool in the bottom of the mouth and the pooled saliva was drained into a collector tube (Salivette; Sarstedt, Nümbrecht, Germany). The samples were centrifuged 2 times at 10,000 g for 300 $\mathrm{s}$, separated into $1 \mathrm{~mL}$ aliquots and frozen at $-70^{\circ} \mathrm{C}$ until required for analysis.

\section{Measurement of Total IgA1 in Saliva and PISF}

Total IgA1 measurement in saliva and PISF were standardized using a capture enzyme-linked immunosorbent assay (ELISA) in accordance with the manufacturer's protocols (e-Bioscience, San Diego, CA, USA). Jackfruit lectin (jacalin), a molecule with a well-studied property of binding to $\lg \mathrm{A}$, was used to capture $\lg \mathrm{A} 1$ in the solid phase of the assay, according to protocol previously described (5). Absorbance was read at $492 \mathrm{~nm}$ using a micro-plate reader (UVM 340; Asys, Eugendorf, Austria) and the measurement of total IgA 1 in saliva samples was established by comparison with the IgA 1 standard curve.

\section{Statistical Analysis}

Results were analyzed by GraphPad Prism, version 3 for Windows (La Jolla; San Diego, CA, USA). Normality of distribution of IgA levels was checked using the Kolmogorov Smirnov Test. Statistically significant differences between IgA levels from health and mucositis implants were analyzed using Mann-Whitey test for independent samples. The relations between the data were analyzed using Spearman Coefficient. Values with $p<0.05$ were considered statistically significant.

\section{Results}

\section{Clinical Findings}

According to the selection criteria, ten patients showed healthy peri-implant tissue and seventeen showed signs of mucositis. The mucositis group $(3.2 \pm 0.7 \mathrm{~mm})$ showed significantly higher mean probing depth in the implant sites than healthy group $(2.1 \pm 0.5 \mathrm{~mm})(p=0.0002)$. The results are expressed as mean and standard deviation.

\section{IgA1 Levels in Healthy and Inflamed Implants}

At all experimental sites, detectable levels of $\lg A 1$ were available. When comparing the IgA 1 levels in sulcular fluid of healthy group $(26.6 \pm 20.7)$ and mucositis group (25.1 \pm 20.5 ), there were no statistically significant differences 
$(p=0.960$, Fig. 1A). Differences between salivary $\lg A 1$ levels were not statistically significant $(p=0.633$, Fig. 1B) in patients with healthy implants $(821.1 \pm 290.6)$ when compared to those with inflamed ones $(779.8 \pm 401.5)$.

\section{Correlation Between PISF and Salivary IgA1 Levels}

In order to determine the similarities between saliva and PISF, and to evaluate these fluids regarding their potential application for IgA 1 analysis in peri-implant disease status, correlation tests were applied to evaluate salivary and PISF $\lg \mathrm{A} 1$ values obtained in healthy and mucositis groups. In the healthy group, no correlation was observed between IgA1 levels in saliva and in sulcular fluid ( $p=0.579, r=0.201)$. The same was noted in the mucositis group when the $\lg A 1$ levels in saliva were compared to those in sulcular fluid $(p=0.728 ; r=0.091)$.

\section{Correlation Between Clinical Parameters and IgA1 Levels}

The implants in the healthy group, no correlation was found between salivary IgA 1 levels and PD means ( $p=0.607$, $r=0.185$ ). No correlation also was found when comparing the IgA1 levels of the mucositis group in sulcular fluid ¿ $(p=0.826 ; r=-0.058)$ or in saliva $(p=0.722 ; r=-0.0934)$ to the PD means. In this group the number of sites bleeding in each implant was not correlated to IgA1 levels detected in saliva or PISF ( $p>0.05)$.

In the healthy group, the number of faces with $P D=3$ $\mathrm{mm}$ in each implant and the total IgA1 levels detected in PISF ( $p=0.137 ; r=-0.505)$ or saliva $(p=0.208 ; r=-0.436)$ were also evaluated without any correlation. Similarly, in the diseased group, no correlation was demonstrated between IgA1 levels in PISF or in saliva and the number of faces with PD values $>3 \mathrm{~mm}<5 \mathrm{~mm}$ (for PISF $p=0.644$, $r=0.121$; for saliva $p>0.05, r=0.220$ ) in implants that showed at least one face with PD value within this interval. Also, the number of faces with PD values higher than $5 \mathrm{~mm}$, in implants that showed at least one face presenting this probing depth, was not correlated to IgA1 levels (for saliva $p=0.140 ;-0.422$; for PISF $p=0.605 ; r=-0.135$ ).

\section{Discussion}

The present study used traditional clinical parameters to determine the peri-implant status of patients with different dental implant systems $(15,16)$. These parameters were associated with the quantification of the immunological marker of inflammation - IgA1, which was quantified in saliva and sulcular fluid of implants and expressed as total IgA1 levels. However, some considerations regarding the experimental design used in this study deserve attention, such as the use of absorbent paper to collect PISF and the placement of all samples in a single device.

The use of absorbent paper aimed to make the method applicable to any clinical situation (14), since the periopaper often used for this purpose is not easily found. The placement of sulcular fluid obtained from each site in a single tube aimed at simplifying the process into one that can easily be performed in a dental office. In addition, the professional often does not treat the inflamed site, but the inflamed implant. Another point to consider is the probe used. Although pre-calibrated probes allow better standardization and probing depth $(17,18)$, the use of this device is not compatible with the clinical reality in many developing countries. Given that the diagnostic method should be simple and widely applicable, we prefer to use conventional probes by a calibrated examiner. The limitations of manual probing can be suppressed by calibration and operator training, rather than operator
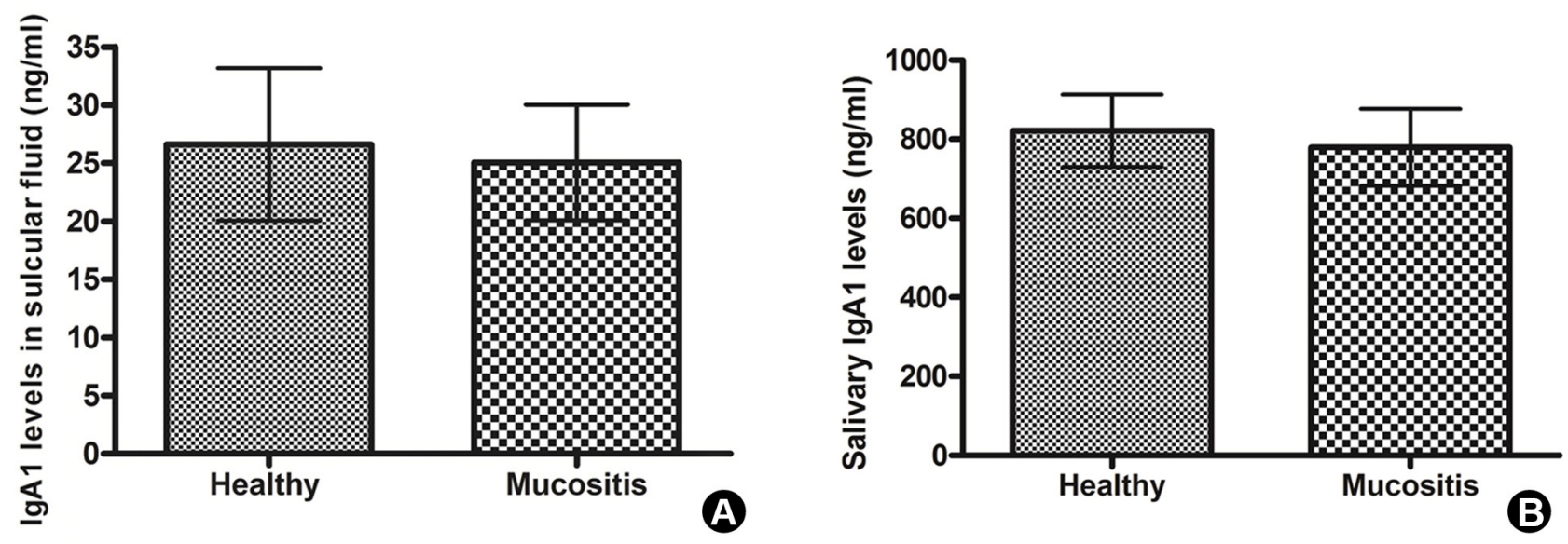

Figure 1. $\lg \mathrm{A} 1$ levels in sulcular fluid (A) and saliva (B) of healthy and inflamed implants, classified as mucositis. $p<0.05$ for differences statistically significant. 
experience (18).

This study analyzes the potential use of $\lg A 1$ as a diagnostic marker of initial peri-implant inflammation. Several studies have already investigated serum antibodies present in saliva and sulcular fluid as indicators or predictors of periodontal status $(2,6)$, although few studies have evaluated their role in peri-implant disease (7). The protective role of IgA in sulcular fluid against periodontal destruction has been attributed to several mechanisms, among them the ability of IgA inhibit bacterial adhesion to the plaque, anti-inflammatory effect by interfering with IgG-mediated complement activation (19), and IgA bonding to specific receptors on polymorphonuclear cells, which could down regulate the activity of these cells (20). In the present study, the mechanisms of periodontal disease are assumed to be the same as those of the peri-implant disease, which lead to evaluation of total IgA1 levels in patients with healthy implants and with peri-implant mucositis.

Although there is no consensus as to the exact mechanism of action of IgA in the pathogenesis of periodontal disease, high levels of salivary IgA directed to specific pathogenic microorganisms have been related to periodontal disease severity $(1,7)$. On the other hand, some studies have reported that patients with gingivitis had a higher concentration of IgA than patients with periodontitis (19). In other words, the concentration of IgA may be elevated in patients with less severe forms of periodontal disease (20). However, in the present study, no differences were observed in total IgA1 levels between healthy and diseased groups, for both saliva and sulcular fluid. A possible explanation for these results is the mild inflammation observed in the inflamed group, in which none of the patients showed signs of peri-implant disease. This is in accordance with the findings of other studies in which there were no differences in antibody levels between healthy and periodontal patients (21), although study designs and patient groups were different.

A greater part of the previous studies using saliva or sulcular fluid investigated IgA levels of specific periodontopathogenic bacteria $(2,6)$, which could influence the host response regarding the bacterial load (22) and specific bacterial proteases $(2,4)$. Though we have no control data on antigen load, it is possible to speculate that the absence of differences between healthy and inflamed implants may have some relationship to the low antigen load in the diseased group, perhaps insufficient to induce a significantly elevated host antibody response (22) to be detected in PISF.

Another explanation for the lack of differences in the $\lg A 1$ response between the studied groups involves specific $\lg \mathrm{A}$ proteases produced by some microorganisms. These proteases are able to induce specific cleavage in $\lg A 1$, but not in IgA2 (2). The inflammation degree can be related to different profiles of subgingival microbiota, leading to differences in host response and consequently in the immunoglobulin profile $(3,6)$.

The present study also compared the IgA1 levels in sulcular fluid and in saliva, and no correlation was found between the immunoglobulin levels in these two sites, for either healthy and diseased implants. Though most diagnostic tests for peri-implantitis have analyzed components of sulcular fluid, the present study also evaluate saliva collected without stimulation as an alternative fluid for this kind of analysis. Saliva samples are advantageous for the screening of periodontal conditions because, when compared to sulcular samples, they are obtained easily and at higher volume (6). Therefore, a possible correlation between IgA 1 levels in this site could substitute the sulcular fluid samples in studies of periodontal and peri-implant disease which was not confirmed in this study.

Patients with peri-implant disease like those with periodontitis would have more sulcular fluid $\lg \mathrm{A}$ and subsequently a higher $\lg \mathrm{A}$ concentration in their saliva than healthy controls (2). However, the present study failed to demonstrate any correlation between either of them. A possible explanation would be the presence of several teeth in the same area as the analyzed implant, which could have contributed to the amount of $\lg A 1$ present in saliva. Clinically healthy teeth are able to produce this immunoglobulin (1) and could contribute to increase these values, leading to a discrepancy in IgA1 levels obtained.

In order to verify the efficacy of sample distribution and to confirm that the absence of differences in IgA1 levels between the groups was not due to the allocation criterion, the clinical parameters, BOP and PD, were correlated to sulcular and salivary IgA 1 levels. However, the results failed to find correlations between IgA 1 levels in the implants and the clinical parameters. The lack of correlation between PD and PISF IgA1 could be explained by the use of mean PD values in the analysis, covering the sites with higher PD. To avoid this kind of interpretation, it was also evaluated the number of faces with PD values $\leq 3 \mathrm{~mm},>3 \mathrm{~mm}<5$ $\mathrm{mm}$, and $>5 \mathrm{~mm}$, which was compared with IgA1 levels in PISF and saliva, maintaining the absence of correlation. These findings are in agreement with those of a periodontal study in which IgA values were independent of the clinical variables and were of no value for diagnostic purposes (23).

Despite the present results, analytical assays based on PISF or saliva sampling are still considered as candidates for the development of a practical predictive diagnostic test for peri-implant disease. Further studies using more sensitive technologies, such as microarray and RT-PCR (20), may reduce the amount of sample need to assays and the variability relative to ELISA methodology (24). 
$\lg$ A1 quantification surrounding the implants could not be used in initial inflammation diagnosis prior to the clinical manifestations of peri-implantitis without further investigations. Due to the important role of $\lg \mathrm{A} 1$ in inflammation, more studies will be necessary to prove its relevance as an initial diagnostic or prognostic marker in peri-implant sites. In fact, the regulation of $\lg A$ response seems to be a complex phenomenon, showing inter-individual differences, and could help explaining the results of the present study.

\section{Resumo}

Não existem estudos que avaliem a utilização de imunoglobulina A1 (IgA1) como marcador precoce da inflamação peri-implantar. 0 objetivo deste estudo foi avaliar os niveis de lgA1 do fluido sulcular peri-implantar (PISF) e saliva de pacientes parcialmente desdentados como indicador da mucosite. Vinte e sete pacientes foram examinados para determinar a condição peri-implantar com base na profundidade de sondagem e sangramento à sondagem. Saliva e PISF ao redor de implantes dentários foram coletados e os niveis IgA1 foram avaliados pelo teste Elisa. IgA1 na saliva e PISF destes pacientes foram comparados e suas correlações com parâmetros clínicos foram avaliados. Não foram observadas diferenças nos niveis de IgA1 $(821,1 \pm 290,6 ; 779,8 \pm 401,5)$ na saliva e PISF $(26,6$ $\pm 20,7 ; 25,1 \pm 20,5)$ de grupos saudáveis e mucosite, respectivamente $(p>0,05)$. Correlação entre os parâmetros clínicos e IgA1 na saliva ou PISF não foi observada em grupos saudáveis ou mucosite $(p=0,607$; $\vec{z} p=0,826$, respectivamente). Estes resultados demonstraram que IgA1 ¿ não pode ser utilizada como marcador imunológico da mucosite.

\section{Acknowledgments}

This research was financially supported by FAPEMIG, Brazil.

\section{References}

1. Kaufman E, Lamster IB. Analysis of saliva for periodontal diagnosis - a review. J Clin Periodontol 2000;27:453-465.

2. Hägewald S, Bernimoulin JP, Köttgen E, Kage A. Salivary IgA subclasses and bacteria-reactive $\lg \mathrm{A}$ in patients with aggressive periodontitis. J Periodontal Res 2002;37:333-339.

3. Ebersole JL. Humoral immune responses in gingival crevice fluid: local and systemic implications. Periodontol 2000 2003;31:135-166.

4. Gomes MA, Rodrigues FH, Afonso-Cardoso SR, Buso AM, Silva AG, Favoreto $S \mathrm{Jr}$, et al.. Levels of immunoglobulin $\mathrm{A} 1$ and messenger RNA for interferon gamma and tumor necrosis factor alpha in total saliva from patients with diabetes mellitus type 2 with chronic periodontal disease. J Periodontal Res 2006;41:177-183.

5. Ozmeric N. Advances in periodontal disease markers. Clin Chim Acta 2004;343:1-16.

6. Fukui $M$, Hinode $D$, Yokoyama $M$, Tanabe $S$, Yoshioka $M$. Salivary immunoglobulin A directed to oral microbial GroEL in patients with periodontitis and their potential protective role. Oral Microbiol Immunol 2006;21:289-295.

7. Kronström M, Svensson B, Erickson E, Houston L, Braham P, Persson GR.
Humoral immunity host factors in subjects with failing or successful titanium dental implants. J Clin Periodontol 2000;27:875-882.

8. Yalçn $S$, Baseğmez C, Mijiritsky E, Yalçn F, Isik G, Onan U. Detection of implant crevicular fluid prostaglandin E2 levels for the assessment of peri-implant health: a pilot study. Implant Dent 2005;14:194-200.

9. Machtei EE, Oved-Peleg E, Peled M. Comparison of clinical, radiographic and immunological parameters of teeth and different dental implant platforms. Clin Oral Implants Res 2006;17:658-665.

10. Karoussis IK, Müller S, Salvi GE, Heitz-Mayfield $\amalg$, Brägger U, Lang NP. Association between periodontal and peri-implant conditions: a 10-year prospective study. Clin Oral Implants Res 2004;15:1-7.

11. Liskmann S, Zilmer M, Vihalemm T, Salum 0, Fischer K. Correlation of peri-implant health and myeloperoxidase levels: a cross-sectional clinical study. Clin Oral Implants Res 2004;15:546-552.

12. Buduneli N, Kinane DF. Host-derived diagnostic markers related to soft tissue destruction and bone degradation in periodontitis. J Clin Periodontol 2011;38:85-105.

13. Hardan S, Khocht A, Suzuki J. A clinical investigation of the association of a biochemical chairside assay and periodontal parameters. J Clin Dent 2011;22:36-39.

14. Perinetti $G$, Spoto $G$. The use of endodontic paper points in determining small fluid volumes. J Applied Res Clinical Dent 2004;1:7-11.

15. Heitz-Mayfield $\amalg$. Peri-implant diseases: diagnosis and risk indicators. J Clin Periodontol 2008;35:292-304.

16. Strbac GD, Monov G, Cei S, Kandler B, Watzek G, Gruber R. Cathepsin $\mathrm{K}$ levels in the crevicular fluid of dental implants: a pilot study. J Clin Periodontol 2006;33:302-308.

17. Gerber JA, Tan WC, Balmer TE, Salvi GE, Lang NP. Bleeding on probing and pocket probing depth in relation to probing pressure and mucosal health around oral implants. Clin Oral Implants Res 2009;20:75-78.

18. Andrade R, Espinoza M, Gómez EM, Espinoza JR, Cruz E. Intra- and inter-examiner reproducibility of manual probing depth. Braz Oral Res 2012;26:57-63.

19. Grbic JT, Lamster IB, Fine JB, Lam KS, Celenti RS, Herrera-Abreu M et al.. Changes in gingival crevicular fluid levels of immunoglobulin $A$ following therapy: association with attachment loss. J Periodontol 1999;70:1221-1227.

20. Gupta G. Gingival crevicular fluid as a periodontal diagnostic indicator - II: Inflammatory mediators, host-response modifiers and chair side diagnostic aids. J Med Life 2013;6:7-13.

21. Graswinckel JE, van der Velden U, van Winkelhoff AJ, Hoek FJ, Loos BG. Plasma antibody levels in periodontitis patients and controls. J Clin Periodontol 2004;31:562-568.

22. Hägewald SJ, Fishel DL, Christan CE, Bernimoulin JP, Kage A. Salivary IgA in response to periodontal treatment. Eur J Oral Sci 2003;111:203208.

23. Hägewald $S$, Bernimoulin JP, Köttgen $E_{1}$ Kage $A$. Total IgA and Porphyromonas gingivalis-reactive $\lg A$ in the saliva of patients with generalised early-onset periodontitis. Eur J Oral Sci 2000;108:147-153.

24. Lamster IB, Ahlo JK. Analysis of gingival crevicular fluid as applied to the diagnosis of oral and systemic diseases. Ann N Y Acad Sci 2007;1098:216-229.
Received April 18, 2013 Accepted July 7, 2013 